\title{
El desafío de reducir los errores de medicación en las Unidades de Cuidado Intensivo Neonatal
}

\author{
The challenge of reducing medication errors in neonatal intensive care units
}

Los errores han estado por siempre presentes en la medicina, y aunque muchos producían daños, incluso la muerte, de ellos no se hablaba ya que prevalecía la cultura de ocultamiento y de castigos para quienes lo cometían. No existían medidas de prevención ni se podía aprender de los errores, elementos esenciales para poder evitarlos. A partir de una investigación comenzó a cambiar la historia y fue un hito histórico "al abrir las puertas" para instalar lo que hoy denominamos seguridad del paciente. El estudio, liderado por el Dr. Leape de la Universidad de Harvard, ${ }^{1}$ mostró al mundo que unos 100000 pacientes hospitalizados fallecían cada año en EE. UU. por errores, (actualmente fallecen más del doble). Luego, fue creciendo en nuestra profesión la conciencia de que era necesario que reconociéramos que todos cometíamos errores en nuestra práctica. Así nacieron los difíciles pasos del cambio de cultura, que aun hoy en día sigue siendo un enorme desafío, que permitió ir abordando relevantes aspectos, el análisis de los errores, las medidas de prevención, el aprender de otras disciplinas de riesgo, diseñar barreras de protección para que el error no llegara al paciente, mejorar la comunicación en los miembros de los equipos hospitalarios y lograr que los pacientes y padres fueran nuestros socios. De esa forma llegamos a estos días con importantes avances pero también con la convicción de que aun nos falta mucho camino por recorrer y que debemos bregar firmemente para que las instituciones médicas puedan alcanzar una seguridad fiable para el bien de los pacientes.

Al menos en nuestro país, sobresalen varios aspectos que influyen para que la cultura de seguridad siga siendo inadecuada. En la profesión médica, así como en enfermería y en todos los que participan en el cuidado de la salud de las personas, no se tiene en cuenta que los errores forman parte de la condición humana y que indefectiblemente van a ocurrir. Asimismo, no fuimos adecuadamente preparados para enfrentar el error, ni en la Universidad y ni en los primeros años de formación, por el contrario continua vigente la falsa suposición que nuestra actividad no tiene que tener errores ya que aun predomina el inapropiado concepto de la infalibilidad.
Respecto al cuidado neonatal, hay acuerdo que es una de las disciplinas médicas más expuesta a errores, en especial en las Unidades de Cuidado Intensivo Neonatal (UCIN). Predominantemente, esto se debe a deficiencias en los sistemas hospitalarios y a la elevada complejidad de los cuidados, motivada en gran parte por la creciente supervivencia de prematuros extremos y de neonatos con malformaciones graves, ambas, poblaciones muy vulnerables. Aun con las diferencias en la prevalencia entre las unidades, se suele considerar a los errores de medicación como los más frecuentemente observados en la $\mathrm{UCIN}^{2,3}$ junto con las infecciones nosocomiales. En EE. UU., los errores de medicación representan unas 700000 visitas a salas de urgencias y más de 100000 hospitalizaciones cada año. Los eventos adversos por drogas (EAD) afectan al 5\% de los pacientes hospitalizados, y es uno de los errores más comunes detectados en hospitales.

La tasa de errores de medicación en la UCIN es muy variable, en un estudio realizado en el Hospital Italiano de Buenos Aires la prevalencia fue de $22 \% .^{2}$ La tasa en la Red Vermon Oxford en EE. UU., fue superior a $40 \% .{ }^{3}$ Un extenso estudio sobre errores en niños, donde el $70 \%$ fueron recién nacidos, se encontró que el 0,6\% presentaron algún evento adverso (EA), 78\% era prevenible y su causa más frecuente (21\%) fueron los errores de medicación. ${ }^{4}$ En un estudio reciente se observó un $49 \%$ de errores de medicación en la UCIN que descendieron a 31\% luego de incrementar la cultura de seguridad. ${ }^{5}$

La tasa de EAD en neonatos es tres veces mayor que en adultos y su mortalidad es más elevada. Esto cobra aún más relevancia ante los resultados irrefutables de varios estudios que nos muestran que los EAD pueden ocasionar a largo plazo trastornos del neurodesarrollo. Este aspecto es mucho más preocupante en los prematuros extremos ya que ciertas drogas aumentarán el ya muy elevado riesgo de posibles secuelas irreparables. ${ }^{6}$

Debemos destacar que estos errores mayoritariamente se relacionan con fallas en el proceso de la medicación, por exceso de confiar en la memoria, y por distracciones en los momentos de la prescripción y la administración. La alta 
frecuencia de errores de medicación en la UCIN están relacionados con varios factores de riesgo. Los principales son: las dosis se calculan por el peso y su gran mayoría deben ser diluidas, casi todos los medicamentos se administran por vía intravenosa, más del 80\% no están aprobados para su uso en RN y las dosis se extrapolan de las indicadas a niños y adultos.

La dilución de medicamentos es uno de los pasos más proclives a que se comentan errores graves, y suele ocurrir cuando debe reiterarse más de una vez, situación no infrecuente, principalmente en prematuros muy pequeños. El error más grave ocurre cuando se administra una dosis 10 veces más alta (o aún mayor) que con bastante frecuencia ocasionan EA graves e incluso la muerte.

Para evaluar porque ocurren los errores de medicación, podemos utilizar el modelo propuesto por Reason en el libro "Human Error", que fue empleado para evaluar las causas de desastres en industrias de alto riesgo y nos permite estudiar el error desde tres perspectivas: el individuo, el entorno inmediato y la cultura organizacional. Extrapolando este modelo a los errores de medicación, los podemos evaluar desde los tres aspectos. El primero es la responsabilidad y capacidad del médico que prescribe y la enfermera/o que administra. Habitualmente, en los hospitales no se les enseña a los médicos residentes, que son los que más prescriben, cómo deben realizar la tarea y tampoco se aplican guías que faciliten su adecuada ejecución. Hay dos premisas importantes que deben enfatizarse para prevenir los errores, ni médicos ni enfermeras/os pueden confiar en su memoria, que es falible en el ser humano y, tanto la prescripción como la preparación del medicamento, deben ser evaluadas por otras personas, para asegurar que son correctas. Esta medida, demostró ser efectiva en múltiples actividades de riesgo, pero lamentablemente, es poco utilizada en medicina. El segundo aspecto ocurre cuando al momento de la prescripción y la administración el entorno es inadecuado. Es frecuente que en las unidades haya múltiples interrupciones y ruidos molestos que afectan las tareas. Un estudio que evaluó 784 errores de medicación, observó que en el 90\% había situaciones que motivaban distracciones en el proceso de la medicación. ${ }^{8}$ El tercero, resulta del nivel de la cultura de seguridad en las instituciones médicas. A mi juicio, es el más importante ya que cambiar la cultura tradicional es muy difícil y mientras eso perdure no se realizará nada de los aspectos que comentamos y no habrá motivación para generar un clima donde la seguridad y la prevención de daños en el paciente sea una de las prioridades esenciales.

Asimismo, quiero reiterar que para disminuir los errores será imprescindible realizar otras acciones sumadas a la que ya vimos, a fin de evitar los EA. Principalmente son, la identificación correcta del paciente, el análisis de las causas de los errores, para aprender de ellos a fin de no reiterarlos, implementar estrategias de prevención y barreras que impidan que el error llegue al paciente, y asimismo, convocar a los padres para que sean nuestros socios a fin de nos ayuden en el arduo desafío de prevenir los errores en sus hijos.

Si en forma responsable cumplimos con las premisas que hemos visto, lograremos alcanzar una mayor seguridad en las unidades neonatales y de esa forma podremos disminuir los eventos adversos en recién nacidos, que sin duda, constituye un imperativo ético ineludible.

\section{José M. Ceriani Cernadas} Editor

http:/ /dx.doi.org/10.5546/aap.2016.394

\section{REFERENCIAS}

1. Brennan TA, Leape LL, Laird NM, Hebert L, et al. Incidence of adverse events and negligence in hospitalized patients. Results of the Harvard Medical Practice Study I. N Engl J Med 1991;324(6):370-6.

2. OteroP,Leyton A,MarianiG,CerianiCernadasJM.Medication errors in pediatric inpatients: prevalence and results of a prevention program. Pediatrics 2008;122(3):e737-43.

3. Suresh G, Horbart JD, Plsek P, Gray J, et al. Voluntary anonymous reporting of medical errors for neonatal intensive care. Pediatrics 2004;113(6):1609-18.

4. Thomas EJ, StuddertDM, Burstin HR, Orav EJ, et al. Incidence and types of adverse events and negligent care in Utah and Colorado. Med Care 2000;38(3):261-71.

5. Chedoe I, Molendijk H, Hospes W, Van den Heuvel ER, et al. The effect of a multifaceted educational intervention on medication preparation and administration errors in neonatal intensive care. Arch Dis Child Fetal Neonatal Ed 2012;97(6):F449-55.

6. Yeh TF, Lin YJ, Lin HC, Huang CC, et al. Outcomes at school age after postnatal dexamethasone therapy for lung disease of prematurity. N Engl J Med 2004;350(13):1304-13.

7. Reason J. Human error: models and management. BMJ 2000;320(7237):768-70.

8. Marino BL, Reinhardt K, Eichelberger WJ, Steingard R. Prevalence of errors in a pediatric hospital medication system: implications for error proofing. Outcomes Manag Nurs Pract 2000;4(3):129-35. 